From the Comprehensive Cancer Center, University of Michigan, Ann Arbor, MI.

Submitted December 8, 2009; accepted March 11, 2010; published online ahead of print at www.jco.org on May 24,2010

Authors' disclosures of potential conflicts of interest and author contributions are found at the end of this article.

Corresponding author: Max S. Wicha, MD, 1500 E Medical Center Dr, 6303 Cancer Center, Ann Arbor, MI 48109 5942; e-mail: mwicha@umich.edu.

(c) 2010 by American Society of Clinical Oncology

0732-183X/10/2825-4006/\$20.00 DOI: $10.1200 / J C 0.2009 .27 .5388$

\title{
Targeting Breast Cancer Stem Cells
}

\author{
Suling Liu and Max S. Wicha
}

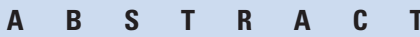

There is increasing evidence that many cancers, including breast cancer, contain populations of cells that display stem-cell properties. These breast cancer stem cells, by virtue of their relative resistance to radiation and cytotoxic chemotherapy, may contribute to treatment resistance and relapse. The elucidation of pathways that regulate these cells has led to the identification of potential therapeutic targets. A number of agents capable of targeting breast cancer stem cells in preclinical models are currently entering clinical trials. Assessment of the efficacy of the agents will require development of innovative clinical trial designs with appropriate biologic and clinical end points. The effective targeting of breast cancer stem cells has the potential to significantly improve outcome for women with both early-stage and advanced breast cancer.

\section{J Clin Oncol 28:4006-4012. (C) 2010 by American Society of Clinical Oncology}

\section{INTRODUCTION}

The past 20 years have seen significant reductions in mortality from breast cancer in the United States and elsewhere. ${ }^{1}$ This reduction has been largely due to improvement in early detection and the development of more effective adjuvant therapies. ${ }^{1}$ In addition, the development of therapies specifically tailored to target each of the molecular subtypes of breast cancer offers new hope for improved survival for patients with metastatic disease. These therapies include human epidermal growth factor receptor 2 (HER2) -targeting agents for HER2-overexpressing tumors, aromatase inhibitors, and third-generation hormonal therapies to target hormone-sensitive disease and poly(ADP-ribose) polymerase (PARP) inhibitors to target BRCA1-deficient and triplenegative breast cancers. ${ }^{2}$ Despite these exciting developments and despite administration of adjuvant therapies, many women still experience relapse, and metastatic breast cancer remains a largely incurable disease. Emerging research suggests that one of the mechanisms accounting for treatment resistance is the existence of subpopulations within human breast cancers that display stem-cell properties. These breast cancer stem cells, by virtue of their relative resistance to conventional therapies, may contribute to treatment resistance and relapse. If this is the case, then the development of strategies to effectively target this cell population may improve patient outcome. In this article, we will review recent progress in the development of biomarkers to identify breast cancer stem cells, the use of model systems to elucidate pathways that regulate these cells, and the development of therapeutic agents to selectively target breast cancer stem-cell populations. We will also discuss the implications of cancer stem-cell models for clinical trial design.

\section{IDENTIFICATION OF BREAST CANCER} STEM CELLS

The cancer stem-cell hypothesis contains two separable but related components. The first, concerning the origin of cancers, holds that cancers arise in cell populations that either maintain or acquire the stem-cell property of self-renewal. The second is that resulting cancers are organized in a hierarchical fashion, in which self-renewing cancer stem cells drive the malignant process as well as generate a population of nonrenewing cells that form the bulk of the tumor.

The development of biomarkers to identify breast cancer stem cells as well as validation of in vitro and mouse models has facilitated the isolation and characterization of these cells from murine and human tumors. The initial description of human breast cancer stem cells involved the prospective isolation of these populations by virtue of their expression of the cell surface markers ESA and CD44 and the absence of expression of the marker CD24. As few as $200 \mathrm{ESA}^{+} / \mathrm{CD}_{4} 4^{+} / \mathrm{CD} 24^{-} / \mathrm{Lin}^{-}$cells were able to generate tumors in immunosuppressed nonobese diabetic/severe combined immunodeficient mice, whereas 100-fold more cells without these markers isolated from the same tumors were nontumorigenic. Furthermore, the tumor-initiating 
population regenerated tumors that recapitulated the heterogeneity of the initial tumor. ${ }^{3}$ More recently, the expression of aldehyde dehydrogenase $(\mathrm{ALDH})$ as assessed by the Aldefluor assay (Stemcell Technologies, Vancouver, Canada) has been used to enrich for tumor-initiating human breast cancer stem-cell populations. Interestingly, these sets of markers identify overlapping but nonidentical cell populations. ${ }^{4}$ Furthermore, there is evidence that different breast cancers may contain tumor-initiating cells that display different cell surface markers. ${ }^{5,6}$ Just as primary human tumors and xenografts have been demonstrated to contain cancer stem-cell populations, established breast cancer cell lines may also contain cellular hierarchies driven by a population expressing cancer stem-cell markers. In addition to tumor initiation, the cells also display increased metastatic potential. ${ }^{5}$ The existence of molecularly characterized cell lines representing the different molecular subtypes of breast cancer has shed light on the potential heterogeneity of breast cancer stem cells. The expression of ALDH in basal but not most luminal cell lines parallels the expression of this marker in human breast cancer subtypes. ${ }^{5}$ This may suggest a different cellular origin of these molecular subtypes. ${ }^{7}$ It is important to emphasize, however, that expression of molecular markers is highly dependent on the microenvironment, necessitating the use of functional assays for cancer stem cells. However, each of these functional assays also has limitations. In vitro assays include the ability of cancer stem cells to survive in anchorage independent conditions to clonally form floating colonies, which have been termed mammospheres, that can be serially passaged. ${ }^{8}$ These in vitro assays have been validated by use of xenotransplantation models that have been considered the gold-standard assay for cancer stem cells. However, increasing evidence suggests an important role for the immune system in regulating cancer behavior pointing to the limitations of xenotransplantation models that utilize immunodeficient mice. Recently, breast cancer stem cells have been isolated from transgenic mouse models. These cells can be serially passaged in the mammary fat pads of syngeneic mice that have intact immune systems, providing a model which more closely simulates human disease. Interestingly, the normal mouse mammary stem cell has been characterized as EpCAM ${ }^{-} / \mathrm{CD}_{4} 9 \mathrm{f}^{+}$. In mouse tumorigenic breast cancers, cells expressing these stemcell markers are tumorigenic when transplanted into immunocompetent syngeneic mice. ${ }^{10}$

\section{BREAST CANCER STEM CELLS AND TREATMENT RESISTANCE}

The identification of cell surface markers for breast cancer stem cells as well as the development of in vitro and mouse models has facilitated studies examining the sensitivity of these cells to various treatment modalities. In vitro studies utilizing established cell lines and primary tumors have suggested that breast cancer stem cells are relatively resistant to radiation therapy and cytotoxic chemotherapy. ${ }^{11}$ The decrease in pro-oxidants in $\mathrm{CD} 44^{+} / \mathrm{CD} 24^{-}$populations may contribute to their radiation therapy resistance. ${ }^{11}$ This resistance may also be mediated by signaling through the Wnt pathway. ${ }^{12}$ The use of neoadjuvant trial design (see Implications of Cancer Stem Cells for Clinical Trial Development) has permitted the clinical validation of the hypothesis that breast cancer stem cells are relatively resistant to cytotoxic chemotherapy. This was accomplished by ascertaining the frequency of cells expressing cancer stem cell markers before and after therapy. When using this approach, it has been reported that the percent of CD $44^{+} / \mathrm{CD} 24^{-}$as well as mammosphere-forming cells increases after administration of neoadjuvant chemotherapy. ${ }^{13}$ Furthermore, the molecular profile of tumors obtained after chemotherapy closely resembles the gene expression profile of untreated CD $44^{+}$/ CD24 $4^{-}$and mammosphere cells. ${ }^{14}$ This profile is also expressed in Claudin ${ }^{\text {low }}$ subset of breast cancers. Interestingly, this gene expression signature is also present in tumors remaining after neoadjuvant endocrine therapy utilizing aromatase inhibitors. ${ }^{14}$ This suggests that hormone-sensitive breast cancers may contain populations of hormone-resistant breast cancer stem cells. The latter observation is supportive of preclinical data suggesting that some estrogen receptor (ER) - expressing breast cancer cell lines contained ER-negative cancer stem cells. ${ }^{15}$ This pattern of expression recapitulates the cellular hierarchy present in the normal mouse and human breast, in which ER-negative stem cells lead to ER-positive luminal cells. ${ }^{16}$ In these tumors, estrogen may indirectly affect ER-negative cancer stem-cell populations through production of paracrine factors by ER-positive bulk tumor cells. These studies have important implications for understanding the mechanisms of treatment resistance to hormone therapies.

In addition to differences in antioxidants, there are a number of molecular mechanisms that may account for the relative resistance of breast cancer stem cells to cytotoxic agents. ${ }^{16}$ These cells are known to express a variety of cellular transporters, including breast cancer resistance protein, a relative of the multidrug resistance protein that can expel chemotherapeutic agents. In fact, increased breast cancer resistance protein expression accounts for the side population, which has been utilized to isolate breast cancer stem cells by flow cytometry. ${ }^{17}$ $\mathrm{ALDH}$, the stem cell marker described in the Identification of Breast Cancer Stem Cells section, is able to inactivate chemotherapeutic agents, such as cyclophosphamide. ${ }^{18}$ Breast cancer stem cells may also express high levels of antiapoptotic proteins, such as survivin and BCL-XL. ${ }^{19}$ In addition, there is evidence for increased efficiency of DNA repair and alterations in cell cycle kinetics in breast cancer stem cells, which may render them relatively resistant to radiation and cytotoxic agents. $^{20}$

\section{TARGETING BREAST CANCER SELF-RENEWAL PATHWAYS}

The relative resistance of breast cancer stem cells to radiation and cytotoxic chemotherapy highlights the need to develop agents able to target this cell population. One of the defining characteristics of cancer stem cells is their ability to undergo self-renewal. During the past decade, a number of developmental pathways that regulate the selfrenewal of normal stem cells have been elucidated. These pathways include Wnt, Notch, and Hedgehog. Interestingly, dysregulation of each of these pathways in the mammary gland generates breast cancers in transgenic mice. ${ }^{21-24}$ Furthermore, there is evidence that these pathways are dysregulated in many human breast cancers. ${ }^{25-27} \mathrm{~A}$ number of excellent reviews describing these pathways have been published, ${ }^{27,28}$ and we will focus on a discussion of issues related to the utilization of these agents in clinical trials. Pathways regulating cancer stem cells and therapeutic agents targeting these pathways are illustrated in Figure 1. 




Fig 1. Targeting signal transduction pathways in breast cancer stem cells. Schematic illustration of key signal transduction pathways, therapeutic targets, and targeting

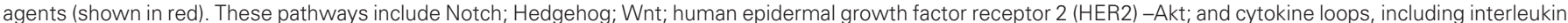
(IL) -6 and IL-8.

There is substantial evidence that the Notch pathway is dysregulated in a substantial fraction of human breast cancers. ${ }^{22,29}$ Furthermore, Notch signaling may play a role in the self-renewal of breast cancer stem cells. Various approaches have been developed to inhibit Notch signaling. The most advanced clinically is the development of $\gamma$ secretase inhibitors. Activation of Notch signaling depends on the proteolytic activity of $\gamma$ secretase, which cleaves Notch receptors releasing their intracellular domains. The $\gamma$ secretase inhibitors initially developed for the treatment of Alzheimer's disease have been administered to patients in early-phase clinical trials. The most significant adverse effect has been gastrointestinal toxicity resulting from goblet cell hyperplasia, an on-target effect of Notch inhibition. ${ }^{30}$ However, this toxicity is schedule dependent, and use of an intermittent schedule, as well as the administration of highdose corticoidsteroids, has reduced this toxicity. ${ }^{31}$ Phase I clinical trials are concurrently underway that combine a $\gamma$ secretase inhibitor with taxane chemotherapy.

Another developmental pathway that regulates breast stem cells is the Hedgehog pathway. This pathway may function directly in tumor cells as well as in the tumor stroma. ${ }^{32}$ Oral Hedgehog inhibitors have entered clinical trials and appear to be relatively nontoxic. ${ }^{33}$
Phase II studies utilizing these compounds in combination with cytotoxic agents are in development.

\section{HER2 SIGNALING AND BREAST CANGER STEM CELLS}

One of the most significant advances in breast cancer therapeutics has been the development of HER2-targeted therapies for the treatment of HER2-overexpressing breast cancers. The addition of HER2-targeting agents, including trastuzumab and lapatinib, to conventional chemotherapy has improved progression-free survival and overall survival of patients with advanced disease. ${ }^{34}$ Moreover, trastuzumab administered in the adjuvant setting has had a significant impact on reducing tumor recurrence. ${ }^{35}$ Recent studies have suggested that the remarkable clinical efficacy of HER2-targeting agents may relate to their ability to target breast cancer stem cells. Studies have demonstrated an association between the expression of the stem cell marker ALDH1 in breast tumors and HER2 amplification. ${ }^{36}$ Furthermore, although neoadjuvant studies using chemotherapy alone have demonstrated that chemotherapy results in a relative increase in the proportion of cancer stem cells, the addition of the HER2-targeting agent lapatinib to chemotherapy reduces this cancer stem-cell population. ${ }^{36}$ This was 
associated with a significantly increased pathologic complete response rate, a clinical end point that has been associated with decreased relapse in other studies. ${ }^{36}$ A molecular explanation for this was suggested by Korkaya et $\mathrm{al}^{36}$ who demonstrated that transfection of HER2 into breast cancer cell lines increased the cancer stem-cell population and resulted in increased invasion and metastasis and that trastuzumab reduced the cancer stem-cell population in HER2overexpressing breast cancer cell lines.

Despite the remarkable clinical efficacy of HER2-targeting agents, one third of HER2-positive tumors do not respond to HER2-targeting agents, and resistance may develop in patients with chronic exposure. Studies have found that nearly $50 \%$ of patients who respond to HER2-targeted agents relapse within a year. ${ }^{37}$ The mechanism of resistance to HER2-targeted agents is not entirely clear. However, increasing evidence indicates that resistance may be associated with loss of the PTEN tumor suppressor gene, gain of function involving somatic mutations of $P I 3 K$, or truncation of the extracellular domain of HER $2 .{ }^{37}$ These mutations result in aberrant activation of the downstream PI3K/Akt pathway and are associated with poor prognosis after trastuzumab therapy. ${ }^{37}$ Indeed, recent evidence has indicated that the PI3K/Akt pathway plays a pivotal role in breast cancer stem-cell regulation. This occurs through Akt activation of the Wnt pathway through phosphorylation and inactivation of GSK $/ 3 \beta$ as well as direct phosphorylation of $\beta$-catenin on serine 552 which facilitates its nuclear transport. ${ }^{38}$ This suggests that inhibiting Akt downstream of HER2 signaling may effectively target breast cancer stem cells in HER2resistant tumors. Indeed, the Akt inhibitor perifosine has been demonstrated to be able to effectively target the breast cancer stem-cell population in breast tumor xenografts. ${ }^{38}$ A number of PI3K and Akt selective inhibitors are currently entering clinical trials allowing for the direct assessment of the effects of these agents on breast cancer stem cells.

\section{TARGETING THE BREAST CANCER STEM} CELL MICROENVIRONMENT

In addition to intrinsic signals regulating breast cancer stem cells, these cells are regulated by elements in the tumor microenvironment. The microenvironment surrounding stem cells has been termed the stemcell niche. In tumors, this niche contains a variety of cellular elements that includes inflammatory cells, fibroblasts, endothelial cells, and mesenchymal stem cells. ${ }^{39}$ Iterative interactions between tumor stem cells, their differentiated progeny, and the microenvironment regulate cellular function through paracrine interactions. Some of these interactions involve signaling pathways described in Targeting Breast Cancer Self-Renewal Pathways, including Wnt, Notch and Hedgehog. In addition, inflammatory cells, fibroblasts, and mesenchymal stem cells may interact with cancer stem cells through cytokine loops. Mesenchymal stem cells may be derived from the normal breast stoma or may be recruited from the bone marrow. ${ }^{40}$ Several inflammatory cytokines, including interleukin IL-6 and IL-8, are demonstrated regulators of breast cancer stem cell self-renewal in in vitro and xenograft models. ${ }^{38,41}$ In addition, chemotherapy-induced cytotoxicity may result in increased local IL- 8 production, which may contribute to the increase in cancer stem-cell populations after chemotherapy. ${ }^{38} \mathrm{Se}$ rum levels of IL- 6 and IL- 8 in patients with advanced breast cancers have been associated with development of metastasis and poor outcome. ${ }^{42-44}$ These cytokines are also increased with chronic inflammation and obesity, conditions associated with increased breast cancer risk. ${ }^{38}$ Interestingly, it has recently been reported that increased serum levels of markers of chronic inflammation, such as C-reactive protein (CRP) and $\beta$-amyloid, are associated with relapse in women with early-stage breast cancer. ${ }^{45-48}$ Because IL- 6 and IL- 8 serum levels are correlated with CRP, the effect of these inflammatory cytokines on breast cancer stem cells might contribute to these clinical observations. These studies suggest that cytokine loops play an important role in regulating cancer stem cells in the cancer stem-cell niche and that developing strategies to interfere with these loops may provide a novel strategy to target cancer stem-cell populations. Interestingly, agents such as statins, which have antiinflammatory effects, have been reported to decrease breast cancer risk. ${ }^{49}$ Statins lower levels of inflammatory cytokines, as reflected by lowered CRP levels. ${ }^{50,51}$ It has also recently been reported that antibodies to the IL- 8 receptor CXCR1 or the small molecule CXCR1/CXCR2 inhibitor repertaxin are to be able to target breast cancer stem cells in xenograft models inhibiting tumor growth and metastasis. ${ }^{38}$ Repertaxin was developed to prevent graft rejection and has been reported to be relatively nontoxic in phase I clinical trials. In addition, monoclonal antibodies targeting IL-6 or its receptor are currently being evaluated in clinical trials for multiple myeloma. ${ }^{52}$ This suggests that agents designed to target inflammatory cytokines in the breast cancer stem-cell niche will be available for clinical testing.

\section{IMPLICATIONS OF CANCER STEM CELLS FOR CLINICAL} TRIAL DEVELOPMENT

The majority of cancer chemotherapeutic agents have been developed by virtue of their ability to cause tumor regression. The efficacy of these agents in clinical trials has been evaluated on the basis of RECIST (Response Evaluation Criteria in Solid Tumors), which reflects tumor size as ascertained by direct measurement or through radiographic imaging. Because tumor size is largely determined by bulk cell populations, it follows that tumor regression largely reflects changes in this population rather than in the rarer cancer stem cells that may drive tumor growth and metastasis. This may explain why, in many solid malignancies including breast cancer, tumor regression does not necessarily translate into increased patient survival. ${ }^{53,54}$ The identification of breast cancer stem-cell biomarkers may allow for direct assessment of therapeutic effects on this cell population. The use of neoadjuvant clinical trial designs in which tumor stem-cell populations can be compared in tumor tissue obtained before and after therapy provides an excellent model to assess activity of breast cancer stem-cell directed therapies. Furthermore, in this setting, complete pathologic response (CPR) is a validated clinical end point, because CPR is associated with reduced recurrence rate. The dramatic increase in CPR rates with addition of HER2-targeting agents in HER2-positive breast cancer supports this concept. ${ }^{13}$

One of the challenges of assessing effects of therapies on breast cancer stem cells is the difficulty of obtaining tissue samples in patients with metastatic disease. Circulating tumor cells (CTCs) may provide a readily obtainable source of such material. However, isolation of breast cancer stem cells from CTCs presents several technical challenges. A number of existing technologies, including the approved Cell 
Search assay (Veridex, Raritan, NJ), use positive selection with EpCAM antibody to isolate CTCs. Because some breast cancer stem cells have been reported to be EpCAM negative, ${ }^{9}$ such assays may miss this cell population. In addition, a number of markers of breast cancer stem cells, including CD44 and ALDH1, are also expressed in hematopoietic stem and progenitor cells, which necessitates the complete elimination of these cell populations before analysis. In spite of these technical challenges, there have been reports of enrichment of breast cancer stem cells in CTC populations compared with matched primary tumors defined by their expression of the markers $\mathrm{CD}_{4} 4^{+} /$ $\mathrm{CD} 24^{-}$or $\mathrm{ALDH} 1^{+}{ }^{55}$ The ability to isolate and access molecular markers in circulating breast cancer stem-cell populations may facilitate pharmacodynamic studies that assess the effects of therapeutic agents on critical cancer stem-cell breast cancer stem-cell regulatory pathways.

\section{ADJUVANT THERAPIES}

Cancer stem-cell models have important implications for the development of adjuvant therapies. In the current paradigm of drug devel- opment, agents that cause tumor shrinkage in the metastatic setting are administered in the adjuvant setting immediately after removal of the primary tumor in an effort to prevent tumor recurrence. This approach is based on the assumption that all cells within a tumor have similar tumor-initiating and metastatic potential; thus, agents that cause tumor regression will also show efficacy in the adjuvant setting. However, as indicated in the Implications of Cancer Stem Cells for Clinical Trial Development section, tumor regression in the advanced setting largely reflects the effect of agents on bulk tumor populations. In contrast, tumor recurrence and metastasis may be driven by the cancer stem-cell population. If this is the case, then agents that target cancer stem cells may be much more effective when administered in adjuvant than in the metastatic setting. This is illustrated graphically in Figure 2A. An agent that only targets cancer stem cells and not bulk tumor cells would be predicted to have only modest effects on tumor size but could have dramatic effects in preventing recurrence in the adjuvant setting. In contrast, agents which primarily affect bulk populations rather than cancer stem cells may cause tumor regression but may be less effective when administered in the adjuvant setting. Bulk tumor cell populations, although not capable of self-renewal, may

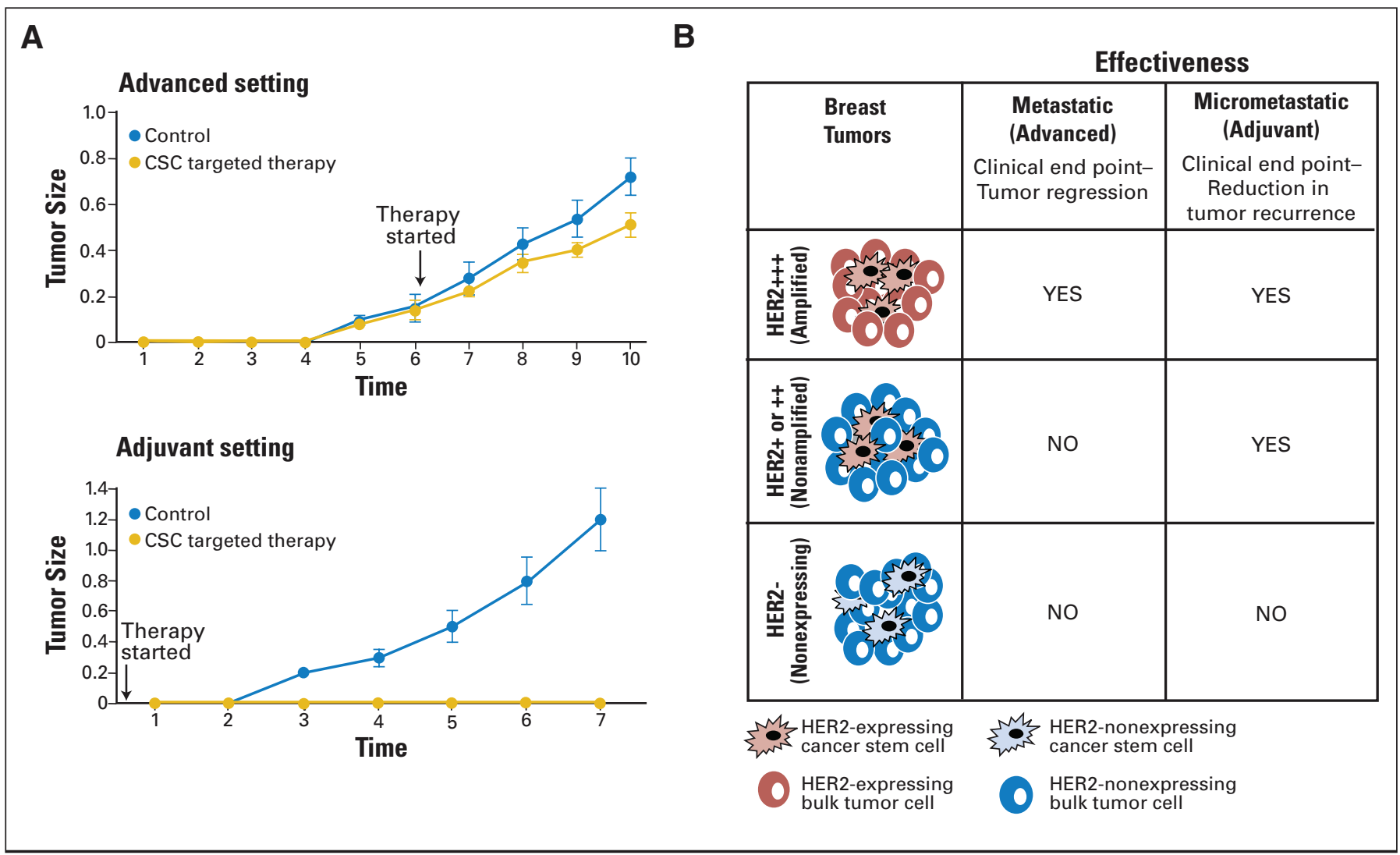

Fig 2. Effects of cancer stem cell (CSC) -targeting agents when administered in advanced versus adjuvant settings. (A) Model illustrating hypothetical effect of

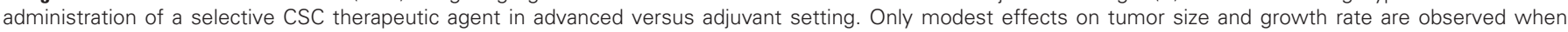

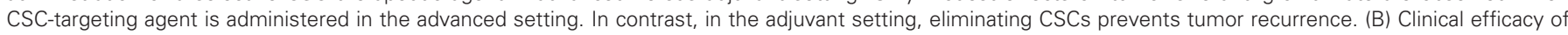

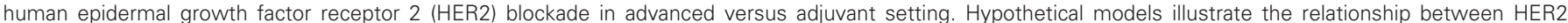

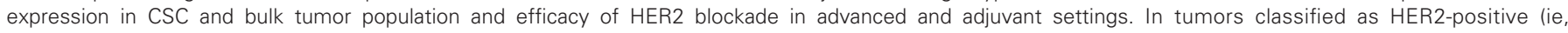

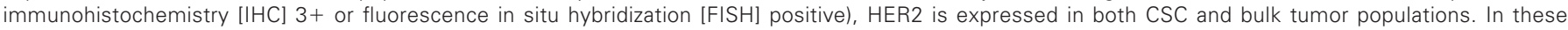

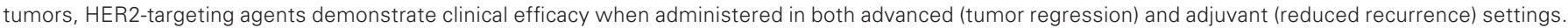

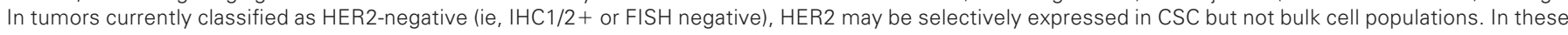

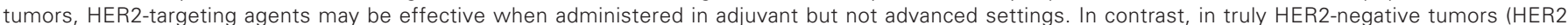
0 by $\mathrm{IHC}$ ), HER2-targeting agents would be predicted to be ineffective in both adjuvant and advanced settings. 
nevertheless be able to undergo a limited number of cell divisions. This may be evaluated as tumor progression even if cancer stem cells have been effectively targeted. This might result in a patient being taken off therapy or a clinical trial being terminated because of tumor progression. If clinical trials only utilize tumor size as an end point, then they may fail to identify effective stem cell-targeting agents. These concepts are illustrated graphically in Figure 2A. The use of CTC-targeting agents in the adjuvant and neodjuvant settings will require judicious monitoring of long-term toxicity in this potentially curable patient population.

The important implications of cancer stem-cell models for patient selection are illustrated by current guidelines regarding selection of patients for therapy with HER2-targeting agents. Currently, use of these agents is limited to patients whose tumors display HER2 amplification as determined by fluorescence in situ hybridization or are $3+$ by immunohistochemistry. This patient selection was based on evidence in metastatic disease that clinical benefit from the HER2targeting agents, such as trastuzumab or lapatinib, occurs primarily in women with HER2-amplified breast cancer. This has resulted in routine testing of breast cancer for HER2 amplification. On the basis of the studies in advanced disease, the addition of HER2-targeting agents in the adjuvant setting has been limited to women whose tumors display HER2 amplification. Recent reports, however, suggest that women with HER2-negative breast cancer who did not meet established criteria for HER2-positive disease on central review received as much clinical benefit from adjuvant trastuzumab as those women with HER2-amplified tumors. ${ }^{56}$ Although these studies require validation by randomized trials, preclinical studies by Magnifico et $\mathrm{al}^{57}$ and others ${ }^{38}$ have suggested a potential explanation for these observations that is consistent with the cancer stem-cell model. In breast cancers without HER2 amplification, HER2 may be selectively expressed in breast cancer stem cells. In these cells, HER2 expression is regulated by Notch signaling. ${ }^{57}$ In these tumors, HER2-targeting agents may selectively target the cancer stem-cell population, which could account for the efficacy of HER2 blockade in the adjuvant setting in tumors without HER2 amplification, in which HER2 is selectively expressed in the cancer stem-cell population (Fig 2B). Furthermore, because HER2 expression is regulated by the Notch pathway, the addition of Notch inhibitors to HER2-targeting agents in this setting represents a rational therapeutic approach (Fig 1).

COMBINATION THERAPIES

We have highlighted the development of therapeutic agents that target cancer stem-cell populations. However, this does not imply that the elimination of bulk tumor populations is not of therapeutic benefit. Indeed, in metastatic disease, symptoms are largely caused by these bulk tumor populations. Thus, tumor regression may be associated with improved quality of life. Furthermore, there is evidence that more differentiated cancer cell populations may acquire cancer stem cell properties through epithelial mesenchymal transition. ${ }^{58,59}$ This highlights the need to target bulk, as well as cancer stem-cell, populations. The combination of cancer stem cell-targeting agents with cytotoxic chemotherapy and/or radiation therapy may provide a means of targeting both cell populations. Furthermore, cancer stemcell populations may evolve during tumor development and therapy, resulting in the generation of multiple cancer stem cell clones within an individual tumor. In addition, cancer stem-cell regulatory pathways are highly interconnected, which suggests that the use of combinations of targeting agents may be necessary to effectively eliminate this cell population. The development of personalized therapy, therefore, may require molecular analysis of cancer stem-cell populations in each patient's tumor. Despite these challenges, the development of stem cell-targeted therapeutics has the potential to significantly improve outcome for patients with both early-stage and advanced breast cancers. As summarized in this review, a number of agents targeting cancer stem-cell pathways are now entering clinical trials. These studies will indicate whether effective targeting of breast cancer stem cells improves patient outcome.

\section{AUTHORS' DISCLOSURES OF POTENTIAL CONFLICTS} OF INTEREST

Although all authors completed the disclosure declaration, the following author(s) indicated a financial or other interest that is relevant to the subject matter under consideration in this article. Certain relationships marked with a " $U$ " are those for which no compensation was received; those relationships marked with a " $C$ " were compensated. For a detailed description of the disclosure categories, or for more information about ASCO's conflict of interest policy, please refer to the Author Disclosure Declaration and the Disclosures of Potential Conflicts of Interest section in Information for Contributors.

Employment or Leadership Position: None Consultant or Advisory Role: Max S. Wicha, OncoMed Pharmaceuticals (C), Pfizer (C) Stock Ownership: Max S. Wicha, OncoMed Pharmaceuticals Honoraria: Max S. Wicha, Pfizer Research Funding: Max S. Wicha, Merck Expert Testimony: None Other Remuneration: None

\section{AUTHOR CONTRIBUTIONS}

Manuscript writing: Suling Liu, Max S. Wicha

Final approval of manuscript: Suling Liu, Max S. Wicha

\section{REFERENCES}

1. Berry DA, Cronin KA, Plevritis SK, et al: Effect of screening and adjuvant therapy on mortality from breast cancer. N Engl J Med 353:17841792, 2005

2. Fong PC, Boss DS, Yap TA, et al: Inhibition of poly(ADP-ribose) polymerase in tumors from BRCA mutation carriers. N Engl J Med 361:123-134, 2009

3. Al-Haji M, Wicha MS, Benito-Hernandez A, et al: Prospective identification of tumorigenic breast cancer cells. Proc Natl Acad Sci U S A 100:39833988, 2003
4. Ginestier $\mathrm{C}$, Hur MH, Charafe-Jauffret $\mathrm{E}$, et al: ALDH1 is a marker of normal and malignant human mammary stem cells and a predictor of poor clinical outcome. Cell Stem Cell 1:555-567, 2007

5. Charafe-Jauffret $E$, Ginestier $C$, lovino F, et al: Breast cancer cell lines contain functional cancer stem cells with metastatic capacity and a distinct molecular signature. Cancer Res 69:1302-1313, 2009

6. Wright $M H$, Calcagno $A M$, Salcido $C D$, et al: Brca1 breast tumors contain distinct CD44+/CD24and CD133+ cells with cancer stem cell characteristics. Breast Cancer Res 10:R10, 2008
7. Dontu G, El-Ashry D, Wicha MS: Breast cancer, stem/progenitor cells and the estrogen receptor. Trends Endocrinol Metab 15:193-197, 2004

8. Dontu G, Abdallah WM, Foley JM, et al: In vitro propagation and transcriptional profiling of human mammary stem/progenitor cells. Genes Dev 17:1253-1270, 2003

9. Shackleton M, Vaillant F, Simpson KJ, et al: Generation of a functional mammary gland from a single stem cell. Nature 439:84-88, 2006

10. Mulholland DJ, Xin L, Morim A, et al: Lin-Sca$1+$ CD49fhigh stem/progenitors are tumor-initiating cells in the Pten-null prostate cancer model. Cancer Res 69:8555-8562, 2009 
11. Dave B, Chang J: Treatment resistance in stem cells and breast cancer. J Mammary Gland Biol Neoplasia 14:79-82, 2009

12. Woodward WA, Chen MS, Behbod F, et al: WNT/beta-catenin mediates radiation resistance of mouse mammary progenitor cells. Proc Natl Acad Sci U S A 104:618-623, 2007

13. Li $X$, Lewis MT, Huang $J$, et al: Intrinsic resistance of tumorigenic breast cancer cells to chemotherapy. J Natl Cancer Inst 100:672-679 2008

14. Creighton CJ, Li X, Landis M, et al: Residua breast cancers after conventional therapy display mesenchymal as well as tumor-initiating features. Proc Natl Acad Sci U S A 106:13820-13825, 2009

15. Horwitz KB, Dye WW, Harrell JC, et al: Rare steroid receptor-negative basal-like tumorigenic cells in luminal subtype human breast cancer xenografts. Proc Natl Acad Sci U S A 105:5774-5779, 2008

16. Asselin-Labat ML, Shackleton M, Stingl J, et al: Steroid hormone receptor status of mouse mammary stem cells. J Natl Cancer Inst 98:1011-1014, 2006

17. Engelmann $K$, Shen $H$, Finn OJ: MCF7 side population cells with characteristics of cancer stem/ progenitor cells express the tumor antigen MUC1. Cancer Res 68:2419-2426, 2008

18. Mirkes PE, Ellison A, Little SA: Role of aldehyde dehydrogenase (ALDH) in the detoxication of cyclophosphamide (CP) in rat embryos. Adv Exp Med Biol 284:85-95, 1991

19. Wang S, Yang D, Lippman ME: Targeting $\mathrm{Bcl}-2$ and $\mathrm{Bcl}-\mathrm{XL}$ with nonpeptidic small-molecule antagonists. Semin Oncol 30:133-142, 2003

20. Frosina G: DNA repair and resistance of gliomas to chemotherapy and radiotherapy. Mol Cancer Res 7:989-999, 2009

21. Vorechovský I, Benediktsson $K P$, Toftgård R: The patched/hedgehog/smoothened signalling pathway in human breast cancer: No evidence for $\mathrm{H} 133 \mathrm{Y}$ $\mathrm{SHH}, \mathrm{PTCH}$ and SMO mutations. Eur J Cancer 35:711-713, 1999

22. Soriano JV, Uyttendaele H, Kitajewski J, et al: Expression of an activated Notch4(int-3) oncoprotein disrupts morphogenesis and induces an invasive phenotype in mammary epithelial cells in vitro. Int J Cancer 86:652-659, 2000

23. Kelly OG, Pinson KI, Skarnes WC: The Wnt co-receptors Lrp5 and Lrp6 are essential for gastrulation in mice. Development 131:2803-2815, 2004

24. Huelsken J, Vogel R, Brinkmann V, et al: Requirement for beta-catenin in anterior-posterior axis formation in mice. J Cell Biol 148:567-578, 2000

25. Pasca di Magliano M, Hebrok M: Hedgehog signalling in cancer formation and maintenance. Nat Rev Cancer 3:903-911, 2003

26. Karhadkar SS, Bova GS, Abdallah N, et al: Hedgehog signalling in prostate regeneration, neoplasia and metastasis. Nature 431:707-712, 2004

27. Liu S, Dontu G, Wicha MS: Mammary stem cells, self-renewal pathways, and carcinogenesis. Breast Cancer Res 7:86-95, 2005
28. Korkaya $H$, Wicha MS: Selective targeting of cancer stem cells: A new concept in cancer therapeutics. BioDrugs 21:299-310, 2007

29. Uyttendaele $H$, Soriano JV, Montesano R, et al: Notch4 and Wnt-1 proteins function to regulate branching morphogenesis of mammary epithelial cells in an opposing fashion. Dev Biol 196:204-217 1998

30. Milano J, McKay J, Dagenais C, et al: Modulation of notch processing by gamma-secretase inhibitors causes intestinal goblet cell metaplasia and induction of genes known to specify gut secretory lineage differentiation. Toxicol Sci 82:341-358, 2004

31. Real PJ, Ferrando AA: NOTCH inhibition and glucocorticoid therapy in T-cell acute lymphoblastic leukemia. Leukemia 23:1374-1377, 2009

32. Tian $\mathrm{H}$, Callahan CA, DuPree KJ, et al: Hedgehog signaling is restricted to the stromal compartment during pancreatic carcinogenesis. Proc Nat Acad Sci U S A 106:4254-4259, 2009

33. Von Hoff DD, LoRusso PM, Rudin CM, et al: Inhibition of the hedgehog pathway in advanced basal-cell carcinoma. N Engl J Med 361:1164-1172, 2009

34. Korkaya $H$, Wicha MS: HER-2, notch, and breast cancer stem cells: Targeting an axis of evil. Clin Cancer Res 15:1845-1847, 2009

35. Mariani G, Fasolo A, De Benedictis E, et al: Trastuzumab as adjuvant systemic therapy for HER2-positive breast cancer. Nat Clin Pract Oncol 6:93-104, 2009

36. Korkaya H, Paulson A, lovino F, et al: HER2 regulates the mammary stem/progenitor cell population driving tumorigenesis and invasion. Oncogene 27:6120-6130, 2008

37. Nagata $Y$, Lan $K H$, Zhou $X$, et al: PTEN activation contributes to tumor inhibition by trastuzumab, and loss of PTEN predicts trastuzumab resistance in patients. Cancer Cell 6:117-127, 2004

38. Korkaya $H$, Paulson A, Charafe-Jauffret $E$, et al: Regulation of mammary stem/progenitor cells by PTEN/Akt/beta-catenin signaling. PLoS Biol 7:e1000121, 2009

39. Albini A, Sporn MB: The tumour microenvironment as a target for chemoprevention. Nat Rev Cancer 7:139-147, 2007

40. Orimo A, Gupta PB, Sgroi DC, et al: Stromal fibroblasts present in invasive human breast carcinomas promote tumor growth and angiogenesis through elevated SDF-1/CXCL12 secretion. Cell 121: 335-348, 2005

41. Sansone P, Storci G, Giovannini C, et al: P66Shc/Notch-3 interplay controls self-renewal and hypoxia survival in human stem/progenitor cells of the mammary gland expanded in vitro as mammospheres. Stem Cells 25:807-815, 2007

42. Inoue K, Slaton JW, Eve BY, et al: Interleukin 8 expression regulates tumorigenicity and metastases in androgen-independent prostate cancer. Clin Cancer Res 6:2104-2119, 2000

43. Inoue $K$, Okabe $S$, Sueoka $E$, et al: The role of interleukin-6 in inhibition of lung metastasis in sub- cutaneous tumor-bearing mice. Oncol Rep 7:69-73, 2000

44. Sansone P, Storci G, Tavolari $S$, et al: IL-6 triggers malignant features in mammospheres from human ductal breast carcinoma and normal mammary gland. J Clin Invest 117:3988-4002, 2007

45. Ridker PM: C-reactive protein and the prediction of cardiovascular events among those at intermediate risk: Moving an inflammatory hypothesis toward consensus. J Am Coll Cardiol 49:2129-2138, 2007

46. Jiralerspong $S$, Angulo AM, Hung MC: Expanding the arsenal: Metformin for the treatment of triple-negative breast cancer? Cell Cycle 8:2681, 2009

47. Jiralerspong S, Palla SL, Giordano SH, et al: Metformin and pathologic complete responses to neoadjuvant chemotherapy in diabetic patients with breast cancer. J Clin Oncol 27:3297-3302, 2009

48. Pierce $B L$, Ballard-Barbash $R$, Bernstein $L$, et al: Elevated biomarkers of inflammation are associated with reduced survival among breast cancer patients. J Clin Oncol 27:3437-3444, 2009

49. Kochhar RKV, Bejjanki $H$, Caldito $G$, et al: Statins reduce breast cancer risk: A case control study in US female veterans. J Clin Oncol 23:6s, 2005 (suppl; abstr 514)

50. Ridker PM: C-reactive protein, inflammation, and cardiovascular disease: Clinical update. Tex Heart Inst J 32:384-386, 2005

51. Ridker PM, Cannon CP, Morrow D, et al: C-reactive protein levels and outcomes after statin therapy. New Engl J Med 352:20-28, 2005

52. Kastritis $E$, Charidimou A, Varkaris $A$, et al: Targeted therapies in multiple myeloma. Target Oncol 4:23-36, 2009

53. Huff CA, Matsui W, Smith BD, et al: The paradox of response and survival in cancer therapeutics. Blood 107:431-434, 2006

54. Blagosklonny MV: Why therapeutic response may not prolong the life of a cancer patient: Selection for oncogenic resistance. Cell Cycle 4:16931698, 2005

55. Theodoropoulos PA, Polioudaki H, Agelaki S, et al: Circulating tumor cells with a putative stem cell phenotype in peripheral blood of patients with breast cancer. Cancer Lett 288:99-106, 2010

56. Paik S, Kim C, Wolmark N: HER2 status and benefit from adjuvant trastuzumab in breast cancer. N Engl J Med 358:1409-1411, 2008

57. Magnifico A, Albano L, Campaner $S$, et al: Tumor-initiating cells of HER2-positive carcinoma cell lines express the highest oncoprotein levels and are trastuzumab sensitive. Clinical Cancer Res (in press)

58. Mani SA, Guo W, Liao MJ, et al: The epithelialmesenchymal transition generates cells with properties of stem cells. Cell 133:704-715, 2008

59. Polyak K, Weinberg RA: Transitions between epithelial and mesenchymal states: Acquisition of malignant and stem cell traits. Nat Rev Cancer 9:265-273, 2009 